\title{
Simulation of fixed bed catalytic reactor through adomian decomposition method and reduced differential transform method
}

\author{
A.S.Pundir*, Kailash Singh and Rajeev K. Dohare \\ Department of Chemical Engineering, Malaviya National Institute of Technology, Jaipur, Rajasthan, India
}

\section{(C2016 ACCENTS}

\begin{abstract}
In this study, adomian decomposition method (ADM) and reduced differential transform method (RTDM) were applied to fixed bed catalytic reactor for hydrolysis of ethyl-acetate using amberlyst-15 as a catalyst. The algorithms of ADM and RTDM were introduced briefly and were examined for non-linear system of partial differential equations (PDE) which were involved in the study of fixed bed catalytic reactor. Results obtained by both methods were compared with numerical solution and showed that methods were quit promising for solving such type of non-linear system of PDE.
\end{abstract}

\section{Keywords}

ADM, RTDM, Fixed bed catalytic reactor, Non-linear PDE.

\section{Introduction}

Nonlinear equations are widely used as models to describe complex physical phenomena and have a significant role in several scientific and engineering fields. The reduced differential transform method (RDTM) was first introduced by Keskin to solve linear and nonlinear PDEs that appears in many mathematical physics and engineering applications. The method provides solutions in an infinite series form and the obtained series may converge to a closed form solution if the exact solution exists.

For concrete problems where exact solution does not exist, the truncated series may be used for numerical purposes. For nonlinear models, the RDTM has shown dependable results and gives analytical approximation that converges very rapidly and in some cases gives exact solutions [1].

Adomian decomposition method (ADM) is used to approximate solution of the ODE \& PDE equation if exact solution does not exist. This method first proposed by American mathematician, George Adomian with the aims to solve frontier physical problem, ADM has successfully been applied to solve many types of both linear and non-linear functional equations [2].

*Author for correspondence

131
1.1 Adomian decomposition method

ADM consists of splitting the given equation into linear and nonlinear parts, inverting the order derivative operator contained in the linear operator on both sides [3], identifying the initial boundary conditions and the first terms initial approximations, decomposing the nonlinear function in terms of special polynomials called Adomian's polynomials, and finding the successive terms of the series solution by recurrent relation using Adomian polynomials [1]. In this section, ADM is explained. First consider the nonlinear partial differential equation given in an operator form[4]:

$L_{t} u(x, t)+L_{x} u(x, t)+R(u(x, t))+N(u(x, t))=g(x, t)$,

Where

$\mathrm{L}_{\mathrm{t}}$ : the highest order differential in $\mathrm{t}$

$\mathrm{L}_{\mathrm{x}}$ : the highest order differential in $\mathrm{x}$

$\mathrm{R}$ : contains the remaining linear terms of lower derivative

$\mathrm{N}$ : is an analytic nonlinear term

$\mathrm{g}$ : represent an in-homogeneous term (source term)

Rearrange the equation as

$L_{t} u(x, t)=g(x, t)-L_{x} u(x, t)-R(u(x, t))-N(u(x, t))$

Applying $\mathrm{L}_{\mathrm{t}}^{-1}$ of both sides of equation (2)

$u(x, t)=\Phi-L_{t}^{-1} g(x, t)-L_{t}^{-1} L_{x} u(x, t)-L_{t}^{-1} R(u(x, t))-L_{t}^{-1} N(u(x, t))$

Where the function $\Phi$ represents the terms arising from using the given conditions. The adomian decomposition method depend of decomposition the unknown function $\mathrm{u}(\mathrm{x}, \mathrm{t})$ into a sum of infinite 
A.S.Pundir et al.

number of components defined by the decomposition series:

$u(x, t)=\sum_{n=0}^{\infty} u_{n}(x, t)$

and the nonlinear term $\mathrm{N}(\mathrm{u}(\mathrm{x}, \mathrm{t}))$ by

$N(u(x, t))=\sum_{n=0}^{\infty} A_{n}$

Where $A_{n}$ are the Adomian polynomials that can be generated for all forms of nonlinearity term by the relation:

$A_{n}=\frac{1}{n !} \frac{\partial^{n}}{\partial \lambda^{n}}\left[N\left(\sum_{i=0}^{n} \lambda^{i} u_{i}\right)\right]_{\lambda=0}, n=0,1,2,3 \ldots ;$

the component $u_{n}(x, t), n \geq 0$ of the solution $u(x, t)$ can be determined by using the recursive relation:

$u_{0}=\phi-L_{t}^{-1} g(x, t)$

$u_{k+1}=-L_{t}^{-1} L_{x} u_{k}-L_{t}^{-1} R\left(u_{k}\right)-L_{t}^{-1}\left(A_{k}\right), k \geq 0$;

The $n$-term approximant $\mathrm{u}_{\mathrm{n}}(\mathrm{x}, \mathrm{t})$ defined by

$u(x, t)=\sum_{n=0}^{\infty} u_{n}(x, t)$

can be used for numerical approximation if closed form solution does not exist.

\subsection{Reduced differential transform method (RDTM)}

In this section reduced differential transform method to solve PDAEs will be described [5].

Definition 1. If a function $\mathrm{u}(\mathrm{x}, \mathrm{t})$ is analytical and continuously differentiable with respect to time $\mathrm{t}$ and space $\mathrm{x}$ in the domain of interest $\Omega$, then Reduced Differential Transform Method (RDTM)

$U_{k}=\frac{1}{k !}\left[\frac{\partial^{k}}{\partial t^{k}} u(x, t)\right]$ at $t=0, x \rightarrow \Omega$

is the transformed function of $\mathrm{u}(\mathrm{x}, \mathrm{t})$.

Definition 2. The differential inverse transform is defined by

$u(x, t)=\sum_{k=0}^{\infty} U_{k}(x) t^{k}$

Substituting (1) into (2), we deduce that

$$
u(x, t)=\sum_{k=0}^{\infty} \frac{1}{k !}\left[\frac{\mathrm{d}^{k}}{\mathrm{~d} t^{k}} u(x, t)\right]_{t=0} t^{k}
$$

From the above definitions, it is easy to see that the concept of the RDTM is obtained from the power series expansion.

The process of RDTM can be described as follows. (1) Apply the reduced differential transform to the initial conditions.

(2) Apply the reduced differential transform to the PDAE to obtain a recursion system for the unknown function $\mathrm{U}_{0}(\mathrm{x}), \mathrm{U}_{1}(\mathrm{x}) \ldots \ldots$.

(3) Use the transformed initial conditions and solve the recursion system for the unknown functions $\mathrm{U}_{0}(\mathrm{x}), \mathrm{U}_{1}(\mathrm{x}) \ldots \ldots$

(4) Use differential inverse transform formula to obtain an approximate or exact solution for the PDAE. The solutions series obtained from RDTM may have limited regions of convergence, even if we take a large number of terms.

Table 1 Transformed function of RDTM [5]

\begin{tabular}{|c|c|}
\hline Function & Transformed \\
\hline$\alpha u(x, t) \mp \beta v(x, t)$ & $\alpha U_{k}(x) \mp \beta V_{k}(x)$ \\
\hline$u(x, t) v(x, t)$ & $\sum_{r=0}^{k} U_{r}(x) V_{k}-r(x)$ \\
\hline$\frac{\partial}{\partial t}[u(x, t)]$ & $(k+1) U_{k+1}(x)$ \\
\hline$\frac{\partial}{\partial x}[u(x, t)]$ & $\frac{\mathrm{d}}{\mathrm{d} x} U_{k}(x)$ \\
\hline$x^{m} t^{n}$ & $\left\{\begin{array}{cc}x^{m} & k=n \\
0 & k \neq a\end{array}\right.$ \\
\hline $\mathrm{e}^{\lambda t}$ & $\frac{\lambda^{k}}{k !}$ \\
\hline $\sin (\omega t+\alpha)$ & $\frac{\omega^{k}}{k !} \sin \left(\frac{\Pi k}{2}+\alpha\right)$ \\
\hline $\cos (\omega t+\alpha)$ & $\frac{\omega^{k}}{k !} \cos \left(\frac{\Pi k}{2}+\alpha\right)$ \\
\hline
\end{tabular}

\section{Mathematical model}

To study the hydrolysis of ethyl acetate in fixed bed reactor [6] which is shown in Figure 1, a one dimensional model was proposed along with assumptions of neglecting various controlling mechanism such as mass transfer control and internal diffusion control which play a dominant role in the study of hydrolysis reaction. Many models for solidliquid kinetics was proposed and experimentally verified but this study focused only a simple power model which required very less data and easily verified. The reaction system can be represented as

$\mathrm{CH}_{3} \mathrm{COOC}_{2} \mathrm{H}_{5}(\mathrm{~A}, \mathrm{~L})+\mathrm{b} \mathrm{H}_{2} \mathrm{O}(\mathrm{B}, \mathrm{L}) \stackrel{\mathrm{K}}{\longrightarrow} \underset{\mathrm{CH}_{3} \mathrm{COOH}+\mathrm{C}_{2} \mathrm{H}_{5} \mathrm{OH}}{\longrightarrow}$ 
where $\mathrm{b}$ is the stiochiometric coefficient. In the present study two liquid phase reactants (A \& B) react on the surface of solid porous catalyst which is amberlyst-15 has proven its fruitful significance in various esterification and hydrolysis reactions. Literature shows that a very less quantity of work is carried out in hydrolysis of ethyl acetate especially when the reaction is performed inside the fixed bed reactor [7]. Here the first approach was given to study the kinetic of the reaction which occurs at the surface of the solid and try to figure out how much possible error. Secondly, the derived kinetic model was used in the one dimensional unsteady state modeling of the fixed bed reactor. The plug flow reactor's conditions were pre-assumed and checked its applicability.

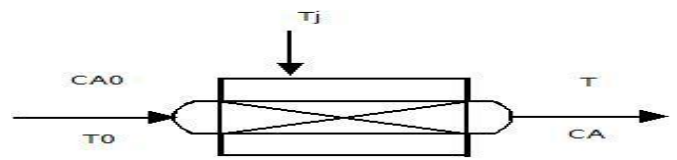

Figure 1 Schematic diagram of fixed bed reactor

$$
\begin{aligned}
& \frac{\partial c_{A}}{\partial t}=-v \frac{\partial c_{A}}{\partial x}-k c_{A} \\
& \frac{\partial T}{\partial t}=-v \frac{\partial T}{\partial x}-\frac{(k \Delta H)}{\left(\rho C_{P}\right)} c_{A}+\frac{A U}{V \rho C_{p}}(T j-T)
\end{aligned}
$$

Initial conditions: $\mathrm{c}_{\mathrm{A}}(\mathrm{x}, \mathrm{t}=0)=\mathrm{c}_{\mathrm{A} 0}, \mathrm{~T}(\mathrm{z}, \mathrm{t}=0)=\mathrm{T}_{0}$

Boundary conditions: $\mathrm{c}_{\mathrm{A}}(\mathrm{x}=0, \mathrm{t})=\mathrm{c}_{\mathrm{A} 1}, \mathrm{~T}(\mathrm{z}=0, \mathrm{t})=\mathrm{T}_{0}$

Transformed the above modelling equations was done into dimensionless form to reduce the complexcity of the system .

$$
\begin{aligned}
& \frac{\partial c_{A}}{\partial t}=-\frac{\partial c_{A}}{\partial x}-q k_{0} \exp (-\gamma / T) c_{A} \\
& \frac{\partial T}{\partial t}=-\frac{\partial T}{\partial x}-\alpha q k_{0} \exp (-\gamma / T) c_{A}+(q / \tau)(T j-T)
\end{aligned}
$$

Write down the equation into the following short notation form

$$
\begin{aligned}
& u_{t}=-u_{x}-q k_{0} \exp \left(-\frac{\gamma}{v}\right) u \\
& v_{t}=-v_{x}-\alpha q k_{0} \exp \left(-\frac{\gamma}{v}\right) u+\frac{q}{\tau}(T j-v) ;
\end{aligned}
$$

\section{Application of ADM}

From the given initial condition, zeroth order term can be selected as

$u_{0}(x, t)=1, v_{0}(x, t)=1$,

For ease drop out the bracket notation $\mathrm{x}, \mathrm{t}$

$u_{0}=1 ; v_{0}=1$;

Taking inverse Operator of equation (20), where

$L_{t}^{-1}()=.\int(.) \mathrm{d} t$

$u_{1}=-L_{t}^{-1}\left[u_{0 x}\right]-k_{0} q L_{t}{ }^{-1}\left(\exp \left(-\frac{\gamma}{v}\right) u\right)$

The non-linear term can be expressed as

$N(u(x, t)$ or $v(x, t))=\sum_{n=0}^{n} A_{n}$

Where $\mathrm{A}_{\mathrm{n}}$ is Adomian polynomial.

$u_{1}=-L_{t}^{-1}\left[u_{0 x}\right]-k_{0} q L_{t}^{-1}\left(A_{0}\right)$;

$A_{0}=\exp \left(-\frac{\gamma}{v_{0}}\right) u_{0}$

$u_{1}=-k_{0} q \exp (-\gamma) t$

Similarly, other dependent variable can be handled

$$
\begin{aligned}
& v_{1}=-L_{t}{ }^{-1}\left[v_{0 x}\right]-\alpha k_{0} q L_{t}{ }^{-1}\left(A_{0}\right)+\frac{q}{\tau}\left(T j-v_{0}\right) \\
& v_{1}=-\alpha k_{0} q \exp (-\gamma) t+\frac{q}{\tau}(T j-1) t \\
& u_{2}=-L_{t}{ }^{-1}\left[u_{1} x\right]-k_{0} q L_{t}^{-1}\left(A_{1}\right) \\
& A_{1}=\exp (-\gamma)\left(\gamma v_{1}+u_{1}\right) \\
& u_{2}=-k_{0} q \exp (-\gamma)\left[\gamma\left\{-k_{0} \alpha q \exp (-\gamma)+\frac{q}{\tau}(T j-1)\right\}\left(\frac{t^{2}}{2}\right)-k_{0} q \exp (-\gamma)\left(\frac{t^{2}}{2}\right)\right] \\
& v_{2}=-k_{0} q \exp (-\gamma)\left[\gamma\left\{-k_{0} \alpha q \exp (-\gamma)+\frac{q}{\tau}(T j-1)\right\}\left(\frac{t^{2}}{2}\right)-k_{0} q \exp (-\gamma)\left(\frac{t^{2}}{2}\right)\right]+\frac{q}{\tau}[T j \cdot t \\
&\left.\quad-k_{0} \alpha q \exp (-\gamma)\left(\frac{t^{2}}{2}\right)-\frac{q}{\tau}(T j-1)\left(\frac{t^{2}}{2}\right)\right]
\end{aligned}
$$

The final form can be written as

$$
\begin{aligned}
& u(x, t)=\sum_{n=0}^{\infty} u_{n}(x, t)=u_{0}+u_{1}+u_{2}+\ldots \ldots \ldots \ldots . . . \\
& v(x, t)=\sum_{n=0}^{\infty} v_{n}(x, t)=v_{0}+v_{1}+v_{2}+\ldots \ldots \ldots \ldots . . .
\end{aligned}
$$

\section{Application of RDTM}

The handling methodology of non-linear term is similar with different approach. The non-linear tern can be written as

$N(u(x, t))=\exp \left(-\frac{\gamma}{v}\right) u=A_{0} \delta(k)+A_{1} \delta(k-1)+A_{2} \delta(k-2)+\ldots .$.

Where

$\delta(k)=\left\{\begin{array}{cc}1, & k=0 \\ 0 & k \neq 0\end{array}\right.$

Taking transformed form of equation (20) and equation (21) with the help of Table 1.

$$
(k+1) U_{k+1}(x)=-\frac{\partial}{\partial x} U_{k}(x)-k_{0} q \exp \left(-\frac{\gamma}{V_{k}(x)}\right) U_{k}(x)
$$


A.S.Pundir et al.

$$
\begin{aligned}
& (k+1) V_{k+1}(x)=-\frac{\partial}{\partial x} V_{k}(x)-k_{0} q \exp \left(-\frac{\gamma}{V_{k}(x)}\right) U_{k}(x)+\frac{q}{\tau}\left(T j-V_{k}\right) \\
& U_{\mathbf{O}}(x)=\mathbf{1} \quad V_{\mathbf{O}}(x)=\mathbf{1}
\end{aligned}
$$

Drop out the bracket notation, can be written as

$$
\begin{aligned}
& U_{\mathbf{0}}=\mathbf{1} V_{\mathbf{O}}=1 \\
& U_{1}=-\frac{\partial}{\partial x} U_{\mathbf{O}}-k_{\mathrm{O}} q \exp \left(-\frac{\gamma}{V_{\mathrm{O}}}\right) U_{\mathrm{O}}=-k_{\mathrm{O}} q \exp (-\gamma) \\
& V_{1}=-\frac{\partial}{\partial x} V_{0}-k_{0} q \exp \left(-\frac{\gamma}{V_{0}}\right) U_{0}+\frac{q}{\tau}\left(T j-V_{0}\right)=-k_{0} q \exp (-\gamma)+\frac{q}{\tau}(T j-1) \\
& 2 U_{2}=-\frac{\partial}{\partial x} U_{1}-k_{0} q \exp (-\gamma)\left(U_{1}+V_{1} \gamma\right)=-k_{0} q \exp (-\gamma)\left(U_{1}+V_{1} \gamma\right) \\
& U_{2}=-k_{0} q \exp (-\gamma)\left(U_{1}+V_{1} \gamma\right)\left(\frac{1}{2}\right) \\
& 2 V_{2}=-\frac{\partial}{\partial x} V_{1}-k_{0} q \exp (-\gamma)\left(U_{1}+V_{1} \gamma\right)+\frac{q}{\tau}\left(T j-V_{1}\right)=-k_{0} q \exp (-\gamma)\left(U_{1}+V_{1} \gamma\right) \\
& \quad+\frac{q}{\tau}\left(T j-V_{1}\right) \\
& V_{2}=\left[-k_{0} q \exp (-\gamma)\left(U_{1}+V_{1} \gamma\right)+\frac{q}{\tau}\left(T j-V_{1}\right)\right]\left(\frac{1}{2}\right) \\
& u(x, t)=\sum_{k=0}^{\infty} U_{k} t^{k}=U_{0}+U_{1} t+U_{2} t^{2}+\ldots \ldots . . \\
& v(x, t)=\sum_{k=0}^{\infty} V_{k^{k}} t^{k}=V_{0}+V_{1} t+V_{2} t^{2}+\ldots \ldots . .
\end{aligned}
$$
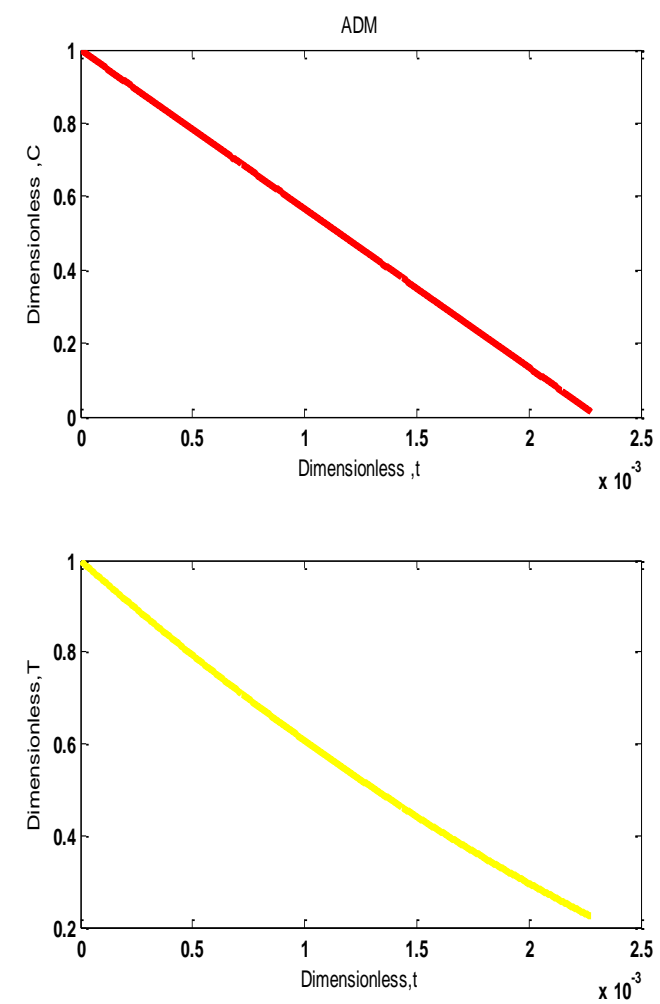

\section{Results \& discussion}

In this paper, fixed bed catalytic reactor was solved with ADM and RDTM methods. Due to non-linear term present, closed form solution did not exist. A plot with two and three terms was plotted for concentration and temperature with respect to dimensionless time to achieve a quit fine accuracy.

Figure 2 shows that the comparison for predicting concentration between the first two/three terms obtained by ADM and RTDM, respectively. These curves show that the both methods have quit good accuracy along with the close resemblance with numerical solution, Figure 4. Similarly, Figure 3 shows that the comparison for predicting temperature between the first two/three terms, However, by considering only first two terms, results were similar in both methods but did not convey clear understanding about the system .To assure this, three terms was plotted and got reliable perdition about the outlet behaviour of dimensionless concentration and temperature with respect to dimensionless time. The accuracy of the proposed methods was checked by numerical results, Figure 4, and got good agreement with these methods.
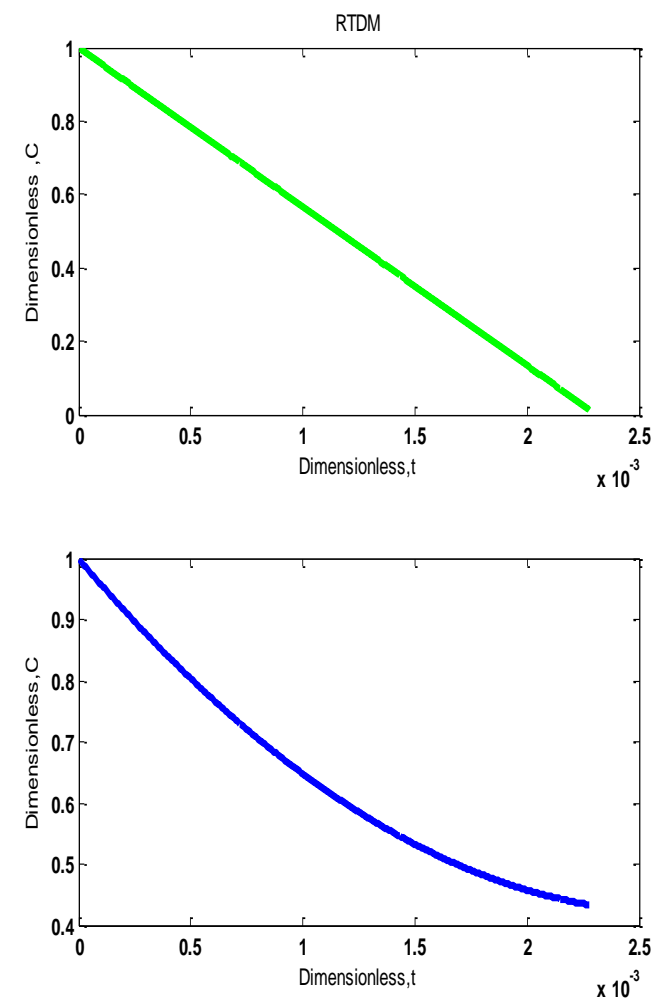

Figure 2 A concentration comparisons between ADM and RDTM for first two / three terms 

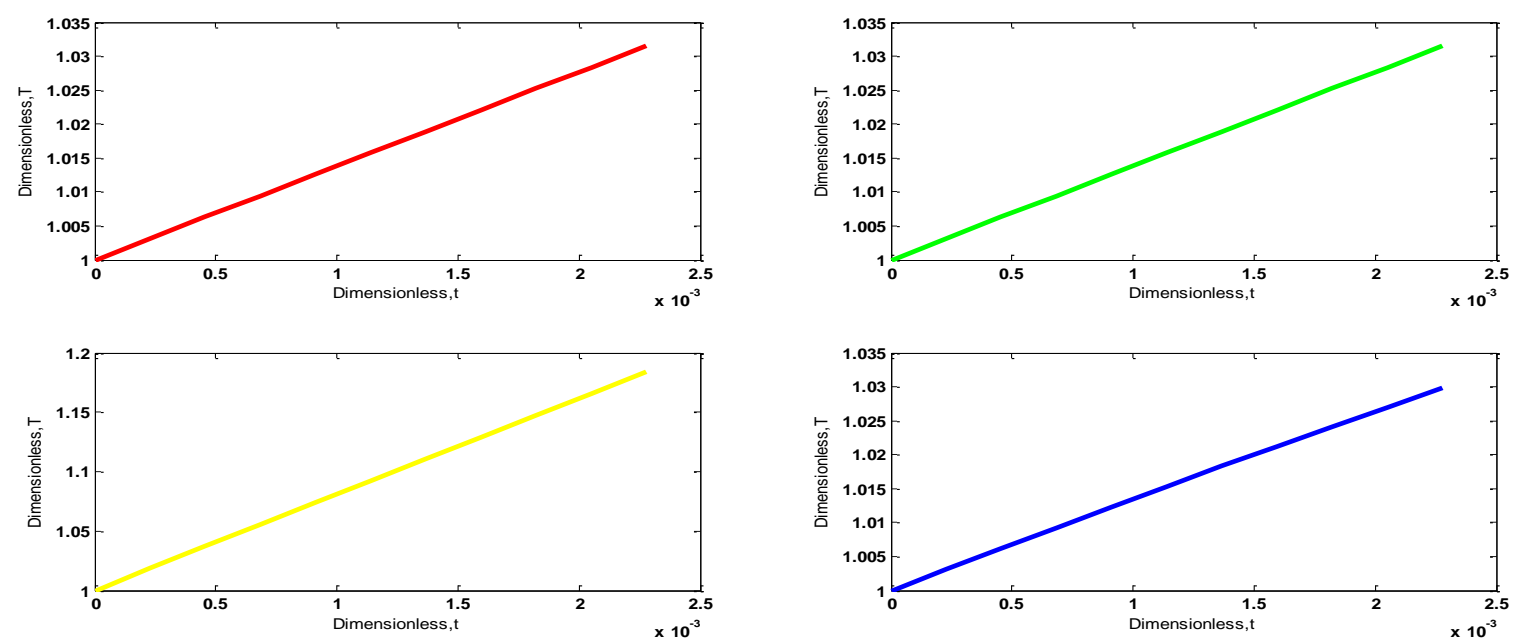

Figure 3 A temperature comparisons between ADM and RDTM for first two / three terms
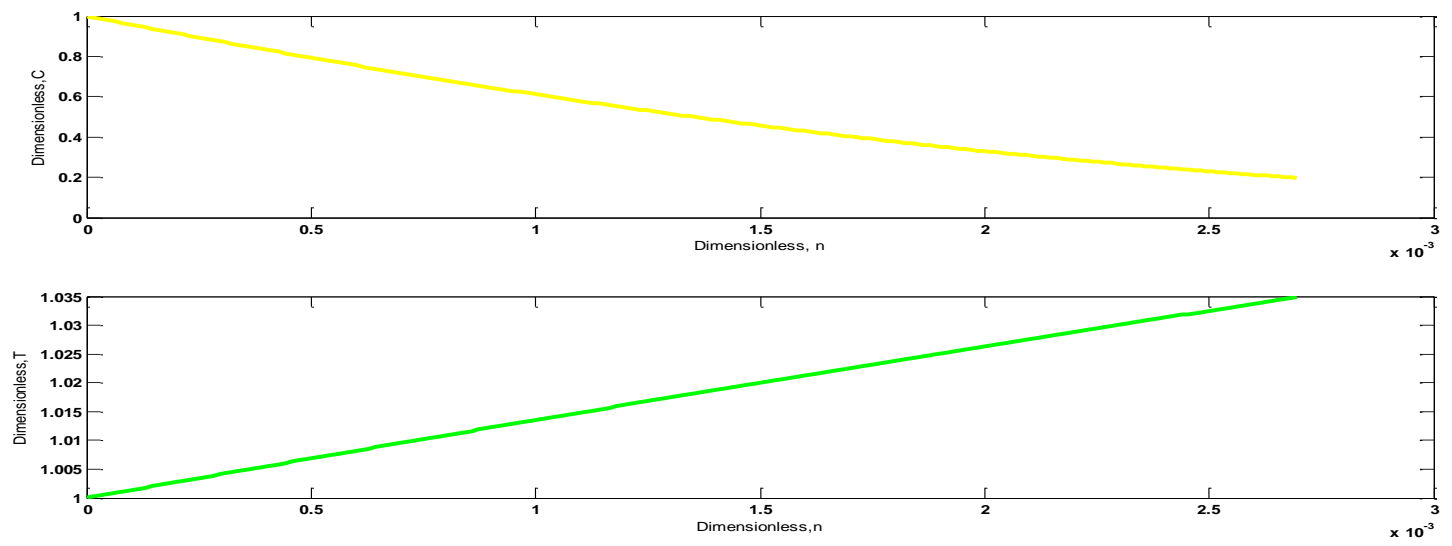

Figure 4 A plots between dimensionless concentration and temperature with respect to dimensionless $n$

\section{Conclusions and future work}

This work presented a comparison between ADM and RDTM methods to solve system of non-linear PDE which often arises in chemical and other engineering field. By solving fixed bed catalytic reactor problem, both methods presented a handy tool with a great potential to solve linear/nonlinear PDEs arise in fixed bed reactor model. It was also shown that if closed form does not exist, which mostly occurs if a non-linear term is present, a more accurate solution can be obtained by retaining few more terms in the series.

\section{Abbreviations}

$\mathrm{t}$ : dimensionless time

$\mathrm{c}_{\mathrm{A}}=\mathrm{u}:$ dimensionless time

$\mathrm{T}=\mathrm{v}:$ dimensionless temperature $\mathrm{q}=$ ratio of velocity and length

$\alpha: \frac{\Delta H T_{O}}{C_{p} O C_{\text {AO }}}$

$\mathrm{k}_{\mathrm{O}}:$ frequency factor

$\gamma$ : Dimensionless activaton energy

$T_{0}$ : initial temperature

$C_{\text {AO }}$ : initial concentration

$\mathrm{x}$ : axial distance

$\tau: \frac{\text { UIIDL }}{\mathrm{VeC}_{\mathrm{p}}}$

$\mathrm{n}: \mathrm{k} * \mathrm{x}+\mathrm{c} * \mathrm{t}$

Acknowledgment

None.

Conflicts of interest

The authors have no conflicts of interest to declare. 


\section{References}

[1] Hosseini SG, Abbasbandy S. Solution of lane-emden type equations by combination of the spectral method and adomian decomposition method. Mathematical Problems in Engineering. 2015.

[2] Jebari R, Ghanmi I, Boukricha A. Adomian decomposition method for solving nonlinear heat equation with exponential nonlinearity. International Journal of Mathematical Analysis. 2013; 7(15):72534.

[3] Al-Mazmumy M, Al-Malki H. Some modifications of adomian decomposition methods for nonlinear partial differential equations. International Journal of Research and Reviews in Applied Sciences. 2015; 23(2):164-73.

[4] Fadugba SE, Zelibe SC, Edogbanya OH. On the adomian decomposition method for the solution of second orders ordinary differential equations. International Journal of Mathematics and Statistics Studies. 2013; 1(2): 20-9.

[5] Benhammouda B, Vazquez-Leal H, Sarmiento-Reyes A. Modified reduced differential transform method for partial differential-algebraic equations. Journal of Applied Mathematics. 2014.

[6] Ayyappan KR, Toor AP, Gupta R, Bansal A, Wanchoo K. Catalytic hydrolysis of ethyl acetate using cation exchange resin (Amberlyst-15): a kinetic study. Bulletin of Chemical Reaction Engineering \& Catalysis. 2009; 4(1):16-22.

[7] Agarwal M, Soni S, Singh K, Chaurasia SP, Dohare RK. Biodiesel yield assessment in continuous-flow reactors using batch reactor conditions. International Journal of Green Energy. 2013; 10(1):28-40.

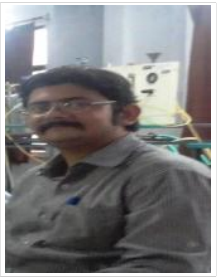

A.S.Pundir was born in Roorkee, India, in 1979. He received the B.Sc degree from the C.C.S University of Meerut, India, in 2001, B.Tech. degree in Chemical Engineering from UPTU, Lucknow in 2005 and the M.Tech. degree in Chemical Engineering from the PUT, Jalandhar, India, in 2013. Currently, he is pursuing PhD from MNIT, Jaipur Email:avdeshpundir@ rediffmail.com

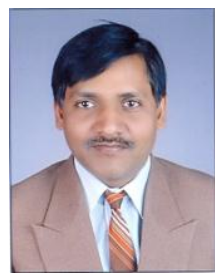

Dr. Kailash Singh pursued his B.E. from University of Roorkee, M. Tech. from IIT Kanpur and Ph.D. from Curtin University of Technology, Australia. Currently, he is working as Associate Professor in Department of Chemical Engineering of Malviya National Institute of Technology, Jaipur (302017), Rajasthan.

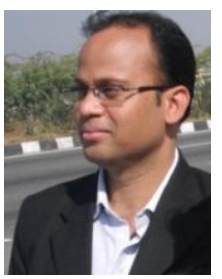

Dr. Rajeev Kumar Dohare pursued his B. Tech. in Chemical Engineering from REC Surat, M.Tech. in Chemical Engineering from AMU Aligarh and Ph.D. in Chemical Engineering from MNIT Jaipur. Currently, he is working as Assistant Professor in Department of Chemical Engineering of Malviya National Institute of Technology, Jaipur (302017), Rajasthan. 